\title{
High Nuclear Expression of Yes-Associated Protein 1 (YAP1) Correlates with Metastasis in Patients with Breast Cancer
}

\section{Yoon Jin Cha}

Gangnam Severance Hospital

Soong June Bae

Gangnam Severance Hospital

Dooreh Kim

Gangnam Severance Hospital

Sung Gwe Ahn

Gangnam Severance Hospital

Joon Jeong

Gangnam Severance Hospital

Ja Seung Koo

Severance Hospital

\section{Tae-Kyung Yoo}

Catholic University of Korea School of Medicine

\section{Woo-Chan Park}

Catholic University of Korea School of Medicine

Ahwon Lee

Catholic University of Korea School of Medicine

Chang Ik Yoon ( $\square$ fayn@daum.net)

St Mary's Hospital, the Catholic University Medical college https://orcid.org/0000-0002-1795-5781

\section{Research article}

Keywords: YAP1, breast cancer, prognosis, TNBC

Posted Date: September 22nd, 2020

DOI: https://doi.org/10.21203/rs.3.rs-77394/v1

License: (1) (1) This work is licensed under a Creative Commons Attribution 4.0 International License.

Read Full License 


\section{Abstract}

Background: Yes-associated protein 1 (YAP1) is a transcription factor regulated by the Hippo pathway and functions as an oncogene in various solid tumors under dysregulated Hippo pathway. However, the role of YAP1 in breast cancer is controversial. Here, we investigated the impact of different levels of nuclear YAP1 expression on the clinical characteristics and survival outcome of patients with breast cancer.

Patients and Methods: Retrospectively obtained 469 breast tumor samples at the Gangnam Severance Hospital were examined for YAP1 expression by immunohistochemistry, and the clinical data were analyzed. External validation was performed using a retrospective cohort and tissues in 489 patients from Severance Hospital.

Results: High nuclear YAP1 expression was associated with hormone receptor negativity and aggressive tumor behavior, including lymph node metastasis, high Ki67 labeling index and inferior distant metastasis-free survival (DMFS), and also confirmed in external validation cohort. In patients with triplenegative breast cancer (TNBC), high nuclear YAP1 expression was an independent significant determinant of poor DMFS (HR 1.947, 95\% Cls 1.003-3.779, P=0.049).

Conclusion: Our findings suggest that nuclear YAP1 expression is a biomarker of adverse prognosis and a potential therapeutic target in patients with breast cancer, especially in TNBC.

\section{Background}

Breast cancer is the most common cancer among women worldwide, and approximately 15 million people are diagnosed with this disease each year. In recent years, owing to advanced treatment modalities and the identification of new drug targets, breast cancer mortality has decreased by approximately $2.3 \%$ per year [1]. Despite these improvements, metastatic breast cancer still results in poor survival. In order to further enhance treatment efficacy, new drug targets with specific roles in metastatic cascades must be unveiled.

Lymph node metastasis is the most important prognostic factor in breast cancer, and is associated with high relapse and mortality [2-5]. The mechanisms underlying lymph node metastasis are still poorly understood. It has been reported that the transcriptional co-activator, Yes-associated protein 1 (YAP1), plays an important role in lymph node metastasis [6]. Enhanced YAP1 activity increases fatty acid oxidation, ultimately leading to lymph node metastasis. YAP1 is controlled by the Hippo pathway and contributes to cancer development by promoting a malignant tumor phenotype. In particular, YAP1 stimulates cancer stem cell proliferation and epithelial-mesenchymal transition, induces drug resistance, inhibits apoptosis, and promotes tumor overgrowth [7-11]. Several studies have reported a correlation of YAP1 expression with aggressive clinical characteristics and low survival [12-22]. This evidence suggests that YAP1 is a potential therapeutic target, and that its pharmacologic or genetic inactivation may suppress tumor progression and improve drug sensitivity. However, the association between clinical 
data and YAP1 expression in patients with breast cancer has been poorly explored. In addition, the possible clinical relevance of YAP1 subcellular localization is not clearly defined.

The aim of this study was to correlation between the level of nuclear YAP1 expression and the clinical characteristics and survival rates of patients with breast cancer. The impact of YAP1 expression on survival was also evaluated in patients with triple negative breast cancer (TNBC).

\section{Materials And Methods \\ 2.1. Patients}

We retrospectively collected tumor tissues from patients undergoing primary curative surgery for breast cancer at the Gangnam Severance Hospital in Seoul, Korea from February 1992 to April 2017 and at the Severance Hospital in Seoul, Korea from January 2000 to December 2010. A validation cohort consisted of 489 patients from the Severance Hospital. All subjects were diagnosed with stage I-III primary breast cancer. All patient treatments were performed according to standard protocols. The following data were collected: age at surgery, tumor size, lymph node status, histological grade (HG), status of estrogen receptor (ER), status of progesterone receptor (PR), status of human epidermal growth factor receptor-2 (HER2), lymphovascular invasion (LVI), Ki67 leveling index, tumor-infiltrating lymphocytes (TILs), treatment modalities, recurrence, and death. Tumor HG was determined by applying the modified ScarffBloom-Richardson grading system. The study protocol was approved by the institutional review board (IRB) of the Gangnam Severance Hospital (local IRB No. 3-2019-0188). The need for informed consent was waived under the approval of the IRB due to the retrospective design.

\subsection{Immunohistochemistry (IHC) and molecular subtyping}

As previously described [23], 3- $\mu$ m thick tissue sections were cut from formalin-fixed paraffin-embedded (FFPE) tissue microarray (TMA) blocks. After deparaffinization and rehydration with xylene and alcohol graded solutions, respectively, IHC was performed by using a Ventana Discovery XT Automated Slide Stainer (Ventana Medical System, Tucson, AZ, USA). Cell Conditioning 1 (CC1) buffer (citrate buffer, pH 6.0; Ventana Medical System) was used for antigen retrieval. The appropriate positive and negative controls were included.

IHC staining was evaluated by light microscopy. Nuclear staining values of $1 \%$ or higher were considered indicative of ER and PR positivity [24]. HER2 staining was interpreted based on the 2018 American Society of Clinical Oncology/College of American Pathologists guidelines [25]. Only samples with strong and circumferential membranous HER2 immunoreactivity (3+) were considered as positive, while those with 0 and $1+$ HER2 staining were regarded as negative. Cases with equivocal HER2 expression (2+) were further evaluated for HER-2 gene amplification by silver in situ hybridization (SISH). Positive nuclear Ki-67 staining was assessed based on the percentage of positive tumor cells, defined as Ki-67 labeling index. 
Breast cancer subcategorization was based on the results of IHC staining for ER, PR, HER2, as well as the SISH results for HER2. The specimens were categorized as follows: i) Luminal/HER2-negative (ER- and/or PR-positive and HER2-negative); ii) HER2-positive (HER2-positive regardless of ER and PR status); iii) TNBC (ER-, PR-, and HER2-negative).

\subsection{Evaluation of nuclear YAP1 expression by tissue microarray and IHC staining}

Hematoxylin and eosin-stained slides from resected breast cancer specimens were examined, and representative areas were marked, and matched tissue cores $(2 \mathrm{~mm})$ were extracted from FFPE tumor blocks and placed into $5 \times 10$ recipient TMA blocks.

For IHC, each TMA slide was stained with an anti-YAP1 antibody (Santa Cruz Biotechnology, Dallas, Texas, USA, 1:200). After staining, nuclear YAP1 expression was assessed by a pathologist using a microscope (400 × magnification). YAP1 expression was evaluated in both the cytoplasm and nuclei of tumor cells. Cytoplasmic staining was evaluated by the $\mathrm{H}$-score, which was obtained by multiplying staining intensity $(0,1,2$ or 3$)$ by percentage of stained area (\%). As nuclear expression was rare and mostly focal, only the intensity of nuclear staining was examined $(0,1+, 2+, 3+)$, regardless of the corresponding cytoplasmic staining. Intensity of nuclear staining of myoepithelial cells was used as an internal control, and assigned to $2+$ value. Weaker and stronger signals were assigned a value of $1+$ and $3+$, respectively. Negative and weak $(1+)$ nuclear staining were considered indicative of low expression, while moderate (2+) and strong (3+) nuclear expression were indicative of high expression (Fig. 3). The $\mathrm{IHC}$ results were interpreted blindly, without any information regarding clinical parameters or outcomes.

\subsection{Statistical analysis}

Distant metastasis-free survival (DMFS) was defined as the period of time between primary curative surgery and the diagnosis of breast cancer-derived distant metastasis, death due to any cause or end of follow-up. Overall survival (OS) was defined as the period from the date of curative primary surgery to the end of follow-up or death due to any cause. Disease-free survival (DFS) was defined as the time to relapse, second cancer, or all cause death, whichever came first. The data of patients who did not exhibit relevant events were censored at the end of follow-up.

Continuous variables between two groups were compared using the Student's t-test or the Mann-Whitney test. Categorical variables were compared by using the Chi-square test or the Fisher's exact test. Survival curves were obtained by the Kaplan-Meier method and two-group comparisons were performed by logrank test. Univariate and multivariate Cox proportional hazard models were used to identify factors associated with survival outcome (DMFS and OS). The variables showing statistically significant differences in the univariate analysis were used in the multivariate Cox proportional hazard models.

Statistical analysis was performed by using SPSS version 24 (SPSS: Chicago, IL, USA) software. The threshold for statistical significance was set at $P<0.05$, with a 95\% confidence interval not including 1 . 


\section{Results}

\subsection{Impact of nuclear YAP1 expression on the baseline characteristics of patients with breast cancer}

A total of 469 breast cancer patients at Gangnam Severance Hospital were included in this study. The median age was 50 (25-86) years. Low and high nuclear YAP1 expression was found in the tumors of $353(75.3 \%)$ and $116(24.7 \%)$ patients, respectively. The clinical characteristics were examined in relation to nuclear YAP1 expression (Table 1). High nuclear YAP1 expression was associated with aggressive tumor features, including hormone receptor negativity, high HG, lymph node metastasis, and high Ki67 expression. Patients were classified into three subtypes based on IHC analysis: luminal/HER2-negative (250 patients), HER2-positive (61 patients), and TNBC (154 patients). High nuclear YAP1 expression was associated with the TNBC subtype. 
Table 1

Clinical characteristics in relation to nuclear YAP1 expression

\begin{tabular}{|c|c|c|c|}
\hline & YAP1-Iow, n = 353 (\%) & YAP1-high, $n=116$ (\%) & $P$ value \\
\hline Age (year, mean $\pm S D$ ) & $50.84 \pm 10.57$ & $49.35 \pm 9.60$ & 0.081 \\
\hline HR & & & $<0.001$ \\
\hline Positive & $243(68.8)$ & $36(31.0)$ & \\
\hline Negative & $110(31.2)$ & $80(69.0)$ & \\
\hline HER2 $^{a}$ & & & 0.245 \\
\hline Positive & $51(14.4)$ & $12(10.3)$ & \\
\hline Negative & $298(84.4)$ & 104 (89.7) & \\
\hline Missing & $4(1.1)$ & 0 & \\
\hline$H^{a}{ }^{a}$ & & & 0.004 \\
\hline I, II & $228(64.6)$ & $56(48.3)$ & \\
\hline III & $119(33.7)$ & $55(47.4)$ & \\
\hline Missing & $6(1.7)$ & $5(4.3)$ & \\
\hline Subtype $^{a}$ & & & $<0.001$ \\
\hline Luminal/HER2(-) & $216(61.2)$ & $34(29.3)$ & \\
\hline HER2 (+) & $49(13.9)$ & $12(10.3)$ & \\
\hline TNBC & $84(23.8)$ & $70(60.3)$ & \\
\hline Missing & $4(1.1)$ & 0 & \\
\hline Tumor size ${ }^{a}$ & & & 0.308 \\
\hline$\leq 2 \mathrm{~cm}$ & $177(50.1)$ & $52(44.8)$ & \\
\hline$>2 \mathrm{~cm}$ & $175(49.6)$ & $64(55.2)$ & \\
\hline Missing & $1(0.3)$ & 0 & \\
\hline Lymph node metastasis $^{\mathrm{a}}$ & & & 0.001 \\
\hline Negative & $238(67.4)$ & $73(62.9)$ & \\
\hline
\end{tabular}

SD, standard deviation; HR, hormone receptor; HER-2, human epidermal growth factor receptor-2; HG, histological grade; TNBC, triple negative breast cancer; TILs, tumor-infiltrating lymphocytes.

aPercentages calculated without missing values 


\begin{tabular}{|c|c|c|c|}
\hline & YAP1-low, n = 353 (\%) & YAP1-high, n = 116 (\%) & $P$ value \\
\hline Positive & $113(32.0)$ & $42(36.2)$ & \\
\hline Missing & $2(0.6)$ & $1(0.9)$ & \\
\hline $\mathrm{Ki} 67(\%)^{\mathrm{a}}$ & & & $<0.001$ \\
\hline$\leq 20 \%$ & $263(74.5)$ & $63(54.3)$ & \\
\hline$>20 \%$ & $78(22.1)$ & $43(37.1)$ & \\
\hline Missing & $12(3.4)$ & $10(8.6)$ & \\
\hline Lymphovascular invasiona & & & 0.536 \\
\hline Negative & 275 (77.9) & 85 (73.3) & \\
\hline Positive & $57(16.1)$ & $21(18.1)$ & \\
\hline Missing & $21(5.9)$ & $10(8.6)$ & \\
\hline TILs $(\%, \text { mean } \pm \text { SD })^{a}$ & $34.47 \pm 26.45(n=169)$ & $33.51 \pm 30.80(n=77)$ & 0.813 \\
\hline \multicolumn{4}{|c|}{$\begin{array}{l}\text { SD, standard deviation; HR, hormone receptor; HER-2, human epidermal growth factor receptor-2; HG, } \\
\text { histological grade; TNBC, triple negative breast cancer; TILs, tumor-infiltrating lymphocytes. }\end{array}$} \\
\hline
\end{tabular}

Validation cohort included 489 patients at Severance Hospital. Of the 489 patients, 434 (88.8\%) had a low nuclear YAP1 expressing tumors, and $55(11.2 \%)$ had high nuclear YAP1 expressing tumors. Clinical characteristics were compared to nuclear YAP1 expression in supplementary table 1 (see additional file 1). Also, high nuclear YAP1 expression was related to TNBC subtype.

\subsection{Prognostic significance of nuclear YAP1 expression}

At a median follow-up time of 59 months (range, 0-325), 42 patients had developed distant metastasis. Among them, 15 had bone metastasis, 11 lung metastasis, 5 liver metastasis, 3 brain metastasis, while 18 had developed metastases to other sites (including duplication). There were 19 mortality events. High nuclear YAP1 expression was significantly associated with decreased distant metastasis-free survival (DMSF) [Fig. 1A; hazard ratio (HR), 2.456, 95\% confidence intervals (Cls) 1.210-4.986, $P=0.0047$, log rank test], and was a significant predictor of poor overall survival (Fig. 1B; HR 4.448, 95\% Cls 1.568$12.62, P=0.0015$, log rank test).

Negative hormone receptor status, tumor size $>2 \mathrm{~cm}$, and high nuclear YAP1 expression were significantly associated with decreased DMFS, as assessed by univariate analysis (Table 2). High nuclear YAP1 expression was still a significant determinant of decreased DMFS after adjustment for hormone receptor 
status, tumor size, and nuclear YAP1 expression by the Cox proportional hazards model (HR 2.216, 95\% Cls $1.130-4.346, P=0.021)$. 
Table 2

Hazard ratios (HRs) and 95\% confidence intervals (Cls) for distant metastasis-free survival (DMFS)

\begin{tabular}{|c|c|c|c|c|}
\hline & Univariate analysis & & Multivariate analysis & \\
\hline & HRs (95\% Cls) & $P$ value & HRs (95\% Cls) & $P$ value \\
\hline Age & $0.979(0.949-1.009)$ & 0.171 & & \\
\hline HR & & 0.010 & & 0.122 \\
\hline Negative & Reference & & Reference & \\
\hline Positive & $0.430(0.227-0.814)$ & & $0.527(0.292-1.157)$ & \\
\hline HER2 & & 0.275 & & \\
\hline Negative & Reference & & & \\
\hline Positive & $0.562(0.200-1.580)$ & & & \\
\hline HG & & 0.070 & & \\
\hline 叉, 囚 & Reference & & & \\
\hline प & $1.751(0.955-3.210)$ & & & \\
\hline Tumor size & & 0.014 & & 0.026 \\
\hline$\leq 2 \mathrm{~cm}$ & Reference & & Reference & \\
\hline$>2 \mathrm{~cm}$ & $2.317(1.184-4.534)$ & & $2.216(1.130-4.346)$ & \\
\hline Lymph node metastasis & & 0.051 & & \\
\hline Negative & Reference & & & \\
\hline Positive & $1.840(0.996-3.397)$ & & & \\
\hline YAP1 expression & & 0.015 & & 0.021 \\
\hline Low & Reference & & Reference & \\
\hline High & $2.145(1.158-3.972)$ & & $2.216(1.130-4.346)$ & \\
\hline Ki67 (\%) & & 0.237 & & \\
\hline$\leq 20 \%$ & Reference & & & \\
\hline$>20 \%$ & $1.530(0.756-3.095)$ & & & \\
\hline Lymphovascular invasion & & 0.391 & & \\
\hline Negative & Reference & & & \\
\hline
\end{tabular}

HR, hormone receptor; HER-2, human epidermal growth factor receptor-2; HG, histologic grade; TILs, tumor-infiltrating lymphocytes. 


\begin{tabular}{|c|c|c|c|c|}
\hline & Univariate analysis & & Multivariate an & \\
\hline & HRs (95\% Cls) & $P$ value & HRs (95\% Cls) & $P$ value \\
\hline Positive & $1.411(0.643-3.099)$ & & & \\
\hline TILs & $0.993(0.977-1.009)$ & 0.405 & & \\
\hline
\end{tabular}

In the univariate Cox proportional hazard model, negative hormone receptor status, high histologic grade, tumor size $>2 \mathrm{~cm}$, and high nuclear YAP1 expression wes found to be significant prognostic factors for OS (Supplementary Table 2, see additional file 1). However, in the multivariate analysis, nuclear YAP1 expression was not a significant predictor of OS (Supplementary Table 2, HR 1.813, 95\% Cls 0.726-4.529, $P=0.203$, see additional file 1 ).

In validation cohort, median follow-up time was 69 months (2-139 months). High nuclear YAP1 expression was significantly predictive of decreased DFS (Supplementary Fig. 1; HR, 2.795, 95\% Cls 1.176-6.644, $P=0.020$, log rank test, see additional file 2). ER negativity, $\mathrm{PR}$ negativity, tumor size $>2 \mathrm{~cm}$, lymph node metastasis, and high nuclear YAP1 expression were significant factors in the multivariate analysis of DFS (supplementary table 3 , see additional file 1 ). When adjusted for other factors, high nuclear YAP1 expression was a significant factor in reduced DFS (HR 1.947, 95\% Cls 1.003-3.779, $P=$ 0.049).

\subsection{Prognostic significance of nuclear YAP1 expression in TNBC patients}

The impact of nuclear YAP1 expression on survival and clinical characteristics was evaluated in patients with TNBC (supplementary Table 4, see additional file 1). Of the 154 TNBC patients, $84(54.5 \%)$ had tumors with low nuclear YAP1 expression, while 70 (45.5\%) had tumors with high nuclear YAP1 expression. In TNBC patients, the clinical characteristics were not significantly affected by the level of nuclear YAP1 expression. However, high nuclear YAP1 expression was associated with poor DMFS (Fig. 2; HR 2.517, 95\% Cls 1.133-5.591, $P=0.0234$, log rank test). Lymph node metastasis and high nuclear YAP1 expression were significant determinants of poor DMFS in the univariate analysis (Supplementary Table 5, see additional file 1). Adjustment for significant factors in the univariate analysis confirmed that high nuclear YAP1 expression was significantly associated with DMFS (HR 2.329, 95\% Cls 1.016-5.339, $P=0.046)$.

\section{Discussion}

In this study, the nuclear expression of YAP1 was evaluated in a large number of breast cancer specimens, was found to be significantly associated with the occurrence of distant metastasis (HR 2.456, $95 \% \mathrm{Cls} 1.210-4.986, P=0.0047)$. Furthermore, nuclear YAP1 expression also was a strong determinant 
of metastasis in TNBC (HR 2.517, 95\%Cls 1.133-5.591, $P=0.0234$ ), an aggressive subtype of breast cancer. These findings indicate that YAP1 is a potential clinical target for the improvement of survival outcomes, particularly DMFS, in patients with breast cancer.

Earlier studies showed that the activation of YAP1, along with that of another transcriptional co-activator (TAZ), is associated with dysregulated Hippo signaling [26, 27]. YAP1 overexpression promotes epithelialmesenchymal transition (EMT), inhibits apoptosis, and induces growth factor-independent cell proliferation. Based on these findings, it was speculated that YAP1 may play a role as a proto-oncogene [16]. In addition, several studies have reported a negative impact of YAP1 activation on the survival of patients with gastric, colorectal, ovarian, bladder, and non-small cell lung cancer [15, 22, 28-30].

The role of YAP1 in breast cancer has been controversial. For instance, Lehn and colleagues reported that YAP1 expression is inversely correlated with HG and tumor cell proliferation, and that low YAP1 mRNA levels are associated with decreased recurrence-free survival and tamoxifen-resistance in luminal $A$ subtype breast cancer [31]. Several other studies have suggested that survival is not significantly affected by YAP1 expression in breast cancer [32,33], or that, on the contrary, YAP1 may act as a tumor suppressor $[19,34]$. Conversely, other studies have reported that YAP1 promotes cell proliferation, tumorigenesis, EMT, and drug resistance, and is associated with P53 mutation, ER negativity, and poor survival $[13,35]$. Among the most of previous studies mentioned above, the specific argument on subcellular localization of YAP1 in previous studies were unclear. Given that nuclear YAP1 expression is a surrogate marker of an activated form of YAP1 [6], we focused on the nuclear YAP1 expression in this study, and found that nuclear YAP1 expression is a strong prognostic predictor for distant metastasis.

Under physiological conditions, the Hippo signaling pathway phosphorylates YAP1 and TAZ and, therefore, their cytosolic localization and ubiquitination [36]. However, dysregulated Hippo signaling results in the nuclear accumulation of non-phosphorylated YAP1 and TAZ. These interact with transcriptional enhanced associated domain (TEAD)-containing transcription factors in nucleus, promoting the expression of genes related to cell proliferation and EMT [37, 38]. Moreover, YAP1 was reported to induce TEAD-dependent focal adhesion kinase phosphorylation, ultimately promoting tumor invasiveness [39].

Our study has several limitations. First, it was a retrospective study. Second, the TNBC subtype was overrepresented due to selection bias during sample collection. Third, TMA slide staining may underestimate the rate of YAP1 positivity compared to whole-slide examination. Although our study is difficult to compare with previous IHC studies [13, 19,31,32,34,40], as these did not clarify whether the employed antibodies were specific for phosphorylated YAP1, nor was the intracellular localization of the antigen established, we specifically examined the nuclear YAP1 expression and confirmed its prognostic effect. In our study, no correlations were found between cytosolic YAP1 staining and survival (data not shown).

Despite these limitations, our study demonstrated that nuclear YAP1 expression was a clinical prognostic factor in breast cancer, especially TNBC.

Page 11/19 


\section{Conclusion}

Our study suggested that nuclear YAP1 expression is a clinical prognostic factor for breast cancer. In addition, YAP1 is a potentially valuable therapeutic target for patients with breast cancer, especially in TNBC.

\section{Abbreviations}

YAP1

Yes-associated protein 1

TNBC

triple negative breast cancer

HG

histological grade

ER

estrogen receptor

PR

progesterone receptor

HER2

human epidermal growth factor receptor-2

LVI

lymphovascular invasion

TILs

tumor-infiltrating lymphocytes

IRB

institutional review board

FFPE

formalin-fixed paraffin-embedded

TMA

tissue microarray

$\mathrm{SISH}$

silver in situ hybridization

DMFS

distant metastasis-free survival

OS

overall survival

DFS

disease free survival

HR

hazard ratio 
Cls

confidence intervals

TAZ

transcriptional co-activator

EMT

epithelial-mesenchymal transition

TEAD

transcriptional enhanced associate domain

\section{Declarations}

\section{Author Contributions:}

Y.J.Cha and C.I.Yoon had full access to all of data in the study and takes responsibility for the integrity of the data and accuracy of the data analysis.

Conceptialization, Y.J.Cha and C.I.Yoon; Data curation, Y.J.Cha, S.J.Bae, D.Kim, J.S.Koo, T.Yoo, W.Park, A.Lee, C.I.Yoon; Funding acquisition, Y.J.Cha ; Investigation, Y.J.Cha, S.J.Bae, D.Kim, S.G.Ahn, J.Jeong, C.I.Yoon ; Methology, Y.J.Cha, C.I.Yoon ; Resources, J.Jeong, J.S.Koo ; Formal analysis, Y.J.Cha, C.I.Yoon ; Supervision, J.Jeong ; Writing-original draft, Y.J.Cha, C.I.Yoon

\section{Funding}

This study was supported by a faculty research grant from the Yonsei University College of Medicine (62018-0080).

\section{Conflict of Interests}

All authors declare that they have no conflict of interest.

\section{Availability of data and materials}

All data generated or analyzed during this study are included in this research article and its additional files.

\section{Ethics approval and consent to participate}

All procedures performed in studies involving human participants were in accordance with the ethical standards of the institutional and/or national research committee and with the 1964 Helsinki Declaration and its later amendments or comparable ethical standards. The protocol was approved by the institutional review board (Local IRB number: 3-2019-0188) of Gangnam Severance Hospital. The need for informed consent was waived under the approval of the IRB due to the retrospective design. 
All authors have given consent for publication.

\section{References}

1. Jemal A, Siegel R, Ward E, Murray T, Xu J, Thun MJ. Cancer statistics, 2007. Cancer J Clin. 2007;57(1):43-66.

2. Ferris RL, Lotze MT, Leong SP, Hoon DS, Morton DL. Lymphatics, lymph nodes and the immune system: barriers and gateways for cancer spread. Clin Exp Metastasis. 2012;29(7):729-36.

3. Carter CL, Allen C, Henson DE. Relation of tumor size, lymph node status, and survival in 24,740 breast cancer cases. Cancer. 1989;63(1):181-7.

4. Atkinson EN, Brown BW, Montague ED. Tumor volume, nodal status, and metastasis in breast cancer in women. J Natl Cancer Inst. 1986;76(2):171-8.

5. SEER Survival Monograph: Cancer Survival Among Adults: US SEER Program, 1988-2001, Patient and Tumor Characteristics. https://seer.cancer.gov/archive/publications/survival/.

6. Lee CK, Jeong SH, Jang C, Bae H, Kim YH, Park I, Kim SK, Koh GY. Tumor metastasis to lymph nodes requires YAP-dependent metabolic adaptation. Science. 2019;363(6427):644-9.

7. Cheng H, Zhang Z, Rodriguez-Barrueco R, Borczuk A, Liu H, Yu J, Silva JM, Cheng SK, Perez-Soler R, Halmos B. Functional genomics screen identifies YAP1 as a key determinant to enhance treatment sensitivity in lung cancer cells. Oncotarget. 2016;7(20):28976-88.

8. Johnson R, Halder G. The two faces of Hippo: targeting the Hippo pathway for regenerative medicine and cancer treatment. Nature reviews Drug discovery. 2014;13(1):63-79.

9. Mo JS, Park HW, Guan KL. The Hippo signaling pathway in stem cell biology and cancer. EMBO Rep. 2014;15(6):642-56.

10. Pan D. The hippo signaling pathway in development and cancer. Developmental cell. 2010;19(4):491-505.

11. Zhao B, Lei QY, Guan KL. The Hippo-YAP pathway: new connections between regulation of organ size and cancer. Curr Opin Cell Biol. 2008;20(6):638-46.

12. Kim DH, Kim SH, Lee OJ, Huang SM, Kwon JL, Kim JM, Kim JY, Seong IO, Song KS, Kim KH. Differential expression of Yes-associated protein and phosphorylated Yes-associated protein is correlated with expression of Ki-67 and phospho-ERK in colorectal adenocarcinoma. Histology histopathology. 2013;28(11):1483-90.

13. Kim SK, Jung WH, Koo JS. Yes-associated protein (YAP) is differentially expressed in tumor and stroma according to the molecular subtype of breast cancer. Int J Clin Exp Pathol. 2014;7(6):322434.

14. Lee KW, Lee SS, Kim SB, Sohn BH, Lee HS, Jang HJ, Park YY, Kopetz S, Kim SS, Oh SC, et al. Significant association of oncogene YAP1 with poor prognosis and cetuximab resistance in colorectal cancer patients. Clinical cancer research: an official journal of the American Association for Cancer Research. 2015;21(2):357-64. 
15. Liu JY, Li YH, Lin HX, Liao YJ, Mai SJ, Liu ZW, Zhang ZL, Jiang LJ, Zhang JX, Kung HF, et al. Overexpression of YAP 1 contributes to progressive features and poor prognosis of human urothelial carcinoma of the bladder. BMC Cancer. 2013;13:349.

16. Overholtzer M, Zhang J, Smolen GA, Muir B, Li W, Sgroi DC, Deng CX, Brugge JS, Haber DA: Transforming properties of YAP, a candidate oncogene on the chromosome 11q22 amplicon. Proceedings of the National Academy of Sciences of the United States of America 2006, 103(33):12405-12410.

17. Wang X, Su L, Ou Q. Yes-associated protein promotes tumour development in luminal epithelial derived breast cancer. European journal of cancer (Oxford England: 1990). 2012;48(8):1227-34.

18. Xu MZ, Yao TJ, Lee NP, Ng IO, Chan YT, Zender L, Lowe SW, Poon RT, Luk JM. Yes-associated protein is an independent prognostic marker in hepatocellular carcinoma. Cancer. 2009;115(19):4576-85.

19. Yuan M, Tomlinson V, Lara R, Holliday D, Chelala C, Harada T, Gangeswaran R, Manson-Bishop C, Smith P, Danovi SA, et al. Yes-associated protein (YAP) functions as a tumor suppressor in breast. Cell death differentiation. 2008;15(11):1752-9.

20. Han SX, Bai E, Jin GH, He CC, Guo XJ, Wang LJ, Li M, Ying X, Zhu Q. Expression and clinical significance of YAP, TAZ, and AREG in hepatocellular carcinoma. Journal of immunology research. 2014;2014:261365.

21. Fitamant J, Kottakis F, Benhamouche S, Tian HS, Chuvin N, Parachoniak CA, Nagle JM, Perera RM, Lapouge M, Deshpande V, et al. YAP Inhibition Restores Hepatocyte Differentiation in Advanced HCC, Leading to Tumor Regression. Cell reports. 2015;10(10):1692-707.

22. Wang Y, Dong Q, Zhang Q, Li Z, Wang E, Qiu X. Overexpression of yes-associated protein contributes to progression and poor prognosis of non-small-cell lung cancer. Cancer Sci. 2010;101(5):1279-85.

23. Yoon Cl, Ahn SG, Bae SJ, Shin YJ, Cha C, Park SE, Lee JH, Ooshima A, Lee HS, Yang KM, et al. High A20 expression negatively impacts survival in patients with breast cancer. PloS one. 2019;14(8):e0221721.

24. Hammond ME, Hayes DF, Dowsett M, Allred DC, Hagerty KL, Badve S, Fitzgibbons PL, Francis G, Goldstein NS, Hayes M, et al. American Society of Clinical Oncology/College Of American Pathologists guideline recommendations for immunohistochemical testing of estrogen and progesterone receptors in breast cancer. Journal of clinical oncology: official journal of the American Society of Clinical Oncology. 2010;28(16):2784-95.

25. Wolff AC, Hammond MEH, Allison KH, Harvey BE, Mangu PB, Bartlett JMS, Bilous M, Ellis IO, Fitzgibbons P, Hanna W, et al. Human Epidermal Growth Factor Receptor 2 Testing in Breast Cancer: American Society of Clinical Oncology/College of American Pathologists Clinical Practice Guideline Focused Update. Journal of clinical oncology: official journal of the American Society of Clinical Oncology. 2018;36(20):2105-22.

26. Kanai F, Marignani PA, Sarbassova D, Yagi R, Hall RA, Donowitz M, Hisaminato A, Fujiwara T, Ito Y, Cantley LC, et al. TAZ: a novel transcriptional co-activator regulated by interactions with 14-3-3 and PDZ domain proteins. EMBO J. 2000;19(24):6778-91. 
27. Sudol M. Yes-associated protein (YAP65) is a proline-rich phosphoprotein that binds to the SH3 domain of the Yes proto-oncogene product. Oncogene. 1994;9(8):2145-52.

28. Jeong W, Kim SB, Sohn BH, Park YY, Park ES, Kim SC, Kim SS, Johnson RL, Birrer M, Bowtell DSL, et al. Activation of YAP1 is associated with poor prognosis and response to taxanes in ovarian cancer. Anticancer research. 2014;34(2):811-7.

29. Song M, Cheong JH, Kim H, Noh SH, Kim H. Nuclear expression of Yes-associated protein 1 correlates with poor prognosis in intestinal type gastric cancer. Anticancer research. 2012;32(9):3827-34.

30. Wang L, Shi S, Guo Z, Zhang X, Han S, Yang A, Wen W, Zhu Q. Overexpression of YAP and TAZ is an independent predictor of prognosis in colorectal cancer and related to the proliferation and metastasis of colon cancer cells. PloS one. 2013;8(6):e65539.

31. Lehn S, Tobin NP, Sims AH, Stål O, Jirström K, Axelson H, Landberg G. Decreased expression of Yesassociated protein is associated with outcome in the luminal $A$ breast cancer subgroup and with an impaired tamoxifen response. BMC Cancer. 2014;14:119.

32. Sheen-Chen SM, Huang CY, Tsai CH, Liu YW, Wu SC, Huang CC, Eng HL, Chan YC, Ko SF, Tang RP. Yes-associated protein is not an independent prognostic marker in breast cancer. Anticancer research. 2012;32(8):3321-5.

33. Vlug EJ, van de Ven RA, Vermeulen JF, Bult P, van Diest PJ, Derksen PW. Nuclear localization of the transcriptional coactivator YAP is associated with invasive lobular breast cancer. Cellular oncology (Dordrecht). 2013;36(5):375-84.

34. Cao L, Sun PL, Yao M, Jia M, Gao H. Expression of YES-associated protein (YAP) and its clinical significance in breast cancer tissues. Human pathology. 2017;68:166-74.

35. Di Agostino S, Sorrentino G, Ingallina E, Valenti F, Ferraiuolo M, Bicciato S, Piazza S, Strano S, Del Sal G, Blandino G: YAP enhances the pro-proliferative transcriptional activity of mutant p53 proteins. EMBO reports 2016, 17(2):188-201.

36. Basu S, Totty NF, Irwin MS, Sudol M, Downward J. Akt phosphorylates the Yes-associated protein, YAP, to induce interaction with 14-3-3 and attenuation of p73-mediated apoptosis. Molecular cell. 2003;11(1):11-23.

37. Zhao B, Kim J, Ye X, Lai ZC, Guan KL. Both TEAD-binding and WW domains are required for the growth stimulation and oncogenic transformation activity of yes-associated protein. Cancer research. 2009;69(3):1089-98.

38. Zhao B, Wei X, Li W, Udan RS, Yang Q, Kim J, Xie J, Ikenoue T, Yu J, Li L, et al. Inactivation of YAP oncoprotein by the Hippo pathway is involved in cell contact inhibition and tissue growth control. Genes Dev. 2007;21(21):2747-61.

39. Shen J, Cao B, Wang Y, Ma C, Zeng Z, Liu L, Li X, Tao D, Gong J, Xie D. Hippo component YAP promotes focal adhesion and tumour aggressiveness via transcriptionally activating THBS1/FAK signalling in breast cancer. Journal of experimental clinical cancer research: CR. 2018;37(1):175. 
40. Jaramillo-Rodríguez Y, Cerda-Flores RM, Ruiz-Ramos R, López-Márquez FC, Calderón-Garcidueñas AL. YAP expression in normal and neoplastic breast tissue: an immunohistochemical study. Arch Med Res. 2014;45(3):223-8.

\section{Figures}

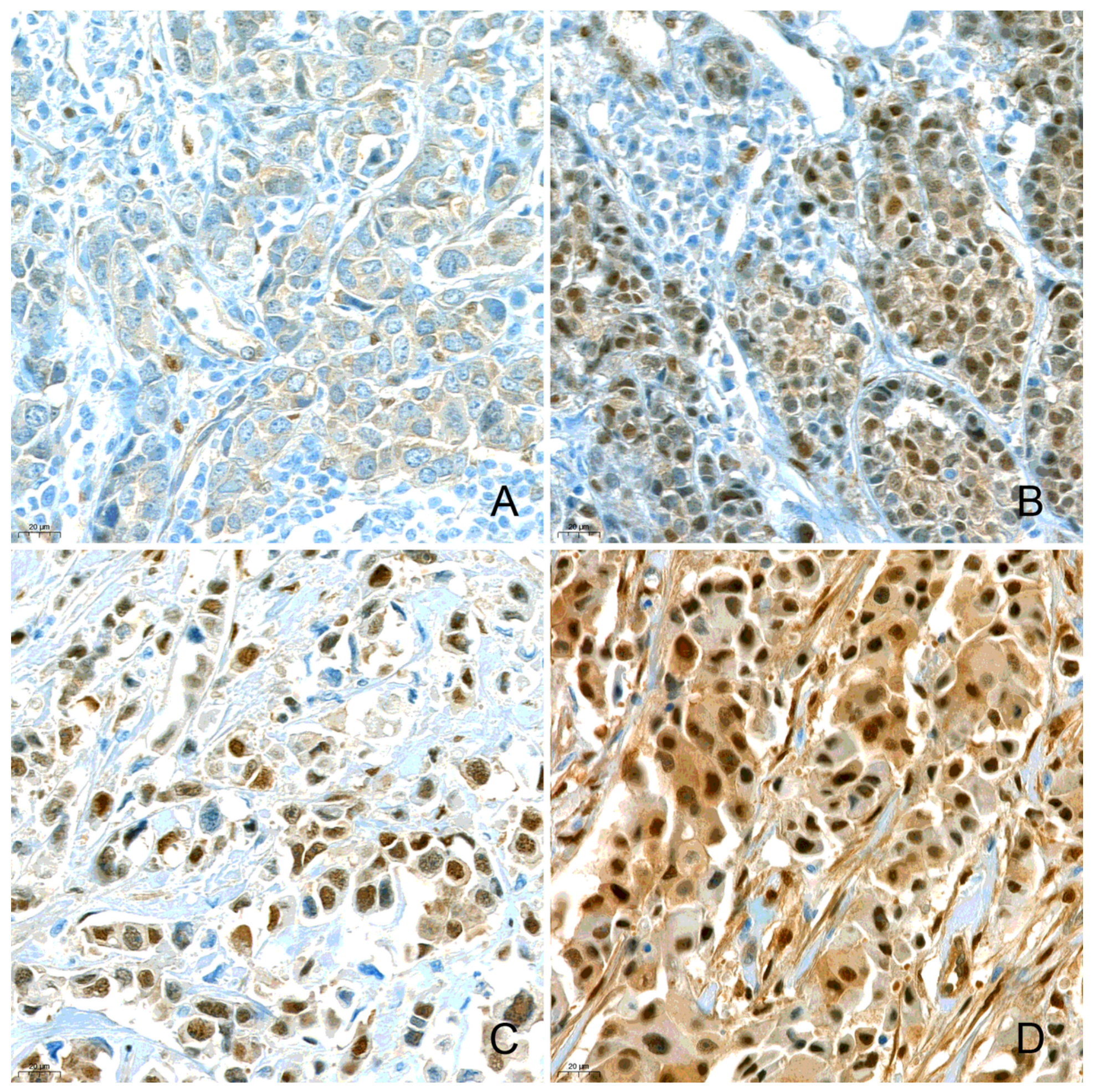

Figure 1 
Immunohistochemical analysis of nuclear YAP1 expression. Nuclear YAP1 expression was evaluated in high-power fields (400囚 magnification) by an experienced pathologist. The samples were classified as negative $(A), 1+(B), 2+(C)$, and $3+(D)$, based on the intensity of YAP1 nuclear staining

(A)

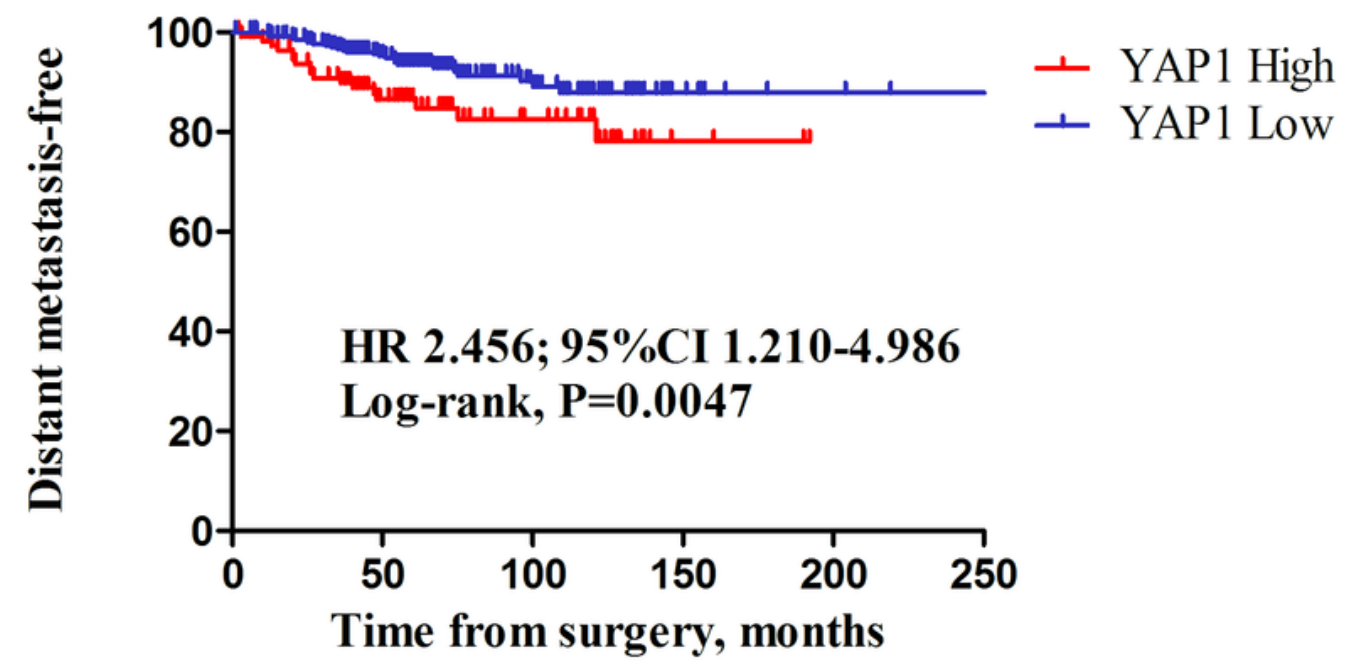

No. at risk

YAP1 Low 353

228

80

10

3

1

YAP1 High 116

72

28

3

0

(B)

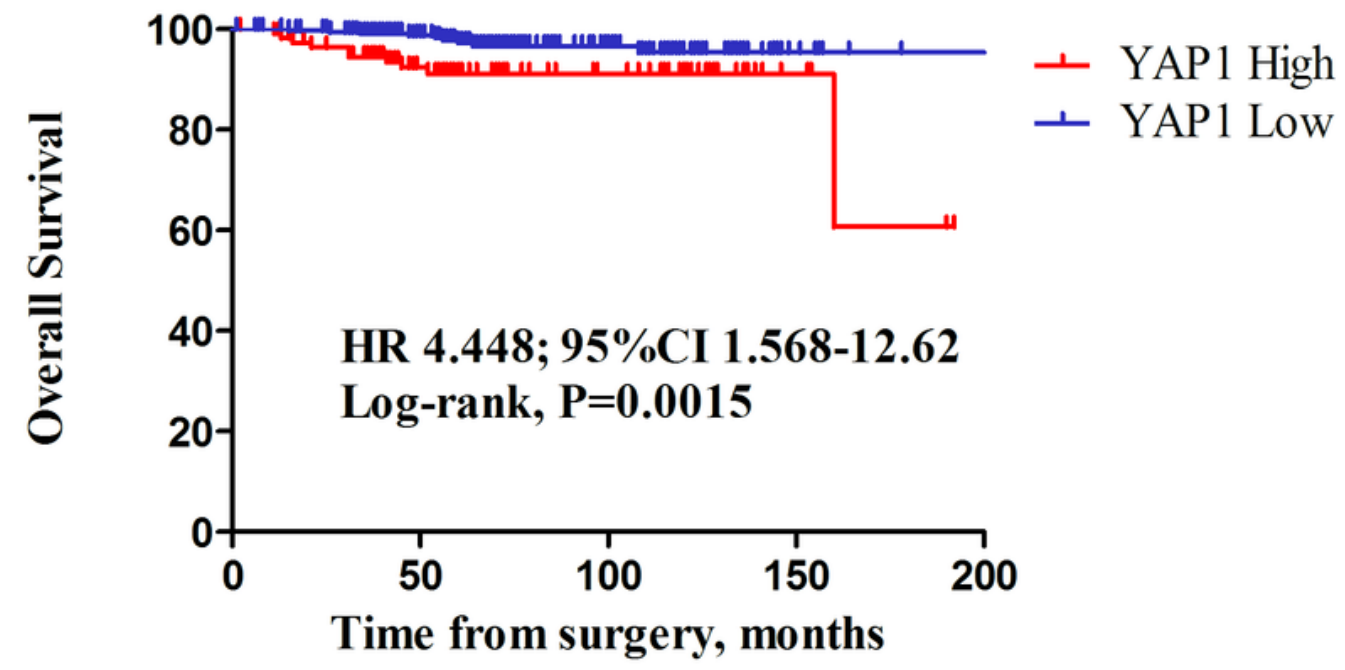

No. at risk

YAP1 Low $\quad 353$

YAP1 High 116

238

75

83

11

3

$\begin{array}{lll}32 & 4 & 0\end{array}$

Figure 2

Kaplan-Meier survival curves of distant metastasis-free survival (DMFS) and overall survival (OS) in relation to nuclear YAP1 expression. Patients with high nuclear YAP1 expression exhibited poor DMFS 
and OS (A, HR 2.456, 95\% Cl 1.210-4.986, $\mathrm{P}=0.0047 ; \mathrm{B}, \mathrm{HR} 4.448,95 \% \mathrm{Cl} 1.568-12.62 ; \mathrm{P}=0.0015$, log-rank test, respectively).

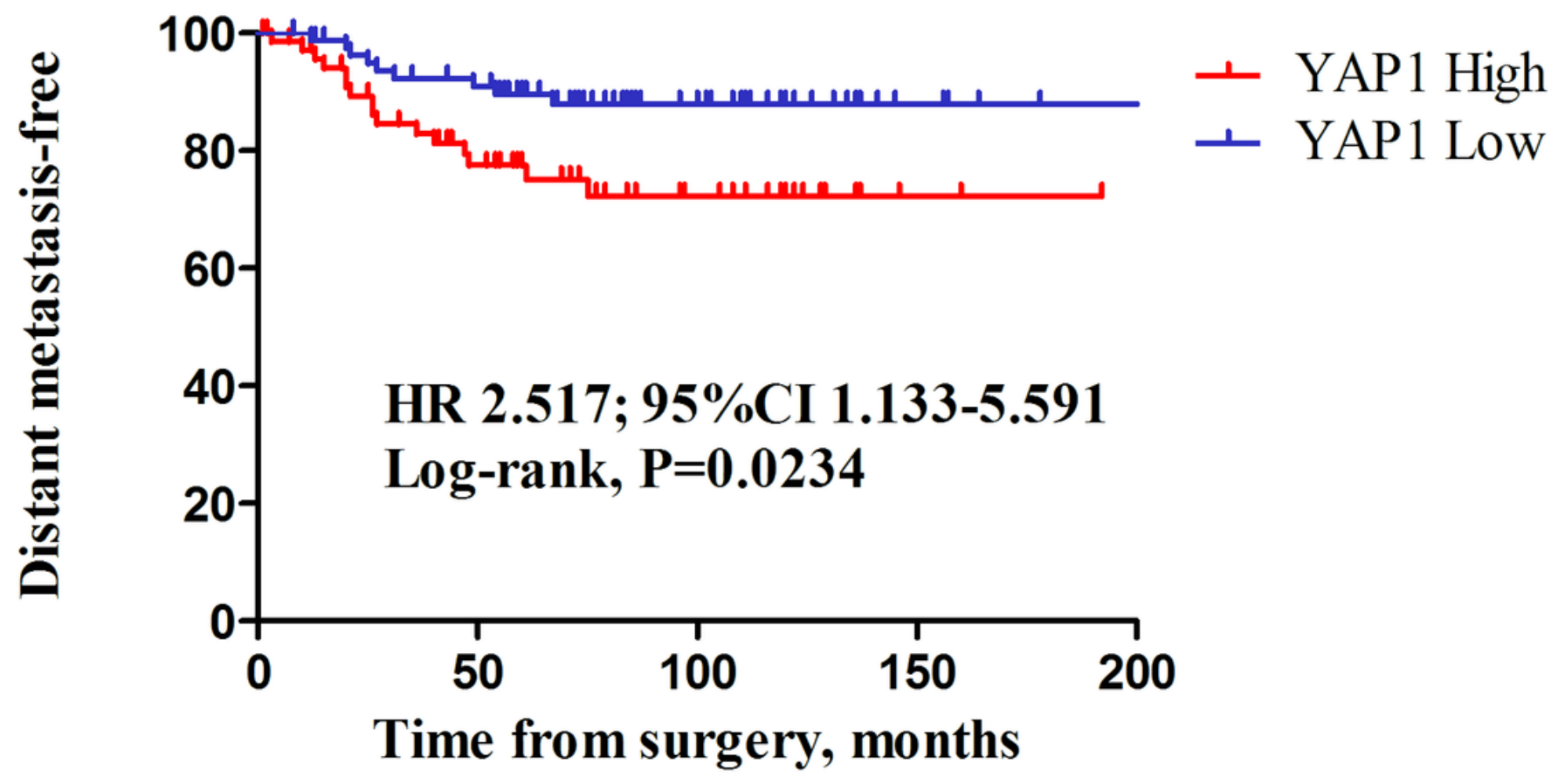

No. at risk

YAP1 Low $\quad 84$

YAP1 High $\quad 70$

68

40

30

18

7

2

0

\section{Figure 3}

Kaplan-Meier survival curves of distant metastasis-free survival (DMFS) in relation to nuclear YAP1 expression in TNBCs. Patients with high nuclear YAP1 expression exhibited poor DMFS (HR 2.517, 95\% Cl 1.133-5.591, $P=0.0234$; log-rank test).

\section{Supplementary Files}

This is a list of supplementary files associated with this preprint. Click to download.

- additionalfile2.tif.tif

- additionalfile1.docx.docx 